3 Dubin J. Tracheotomia. In: Elsevier (Ed.). Encyclopédie Médico-Chirurgicale. Tecniche chirurgiche. Chirurgia ORL e cervicofacciale. Paris, France. 1995: 46-430.

4 Agrò F, Hung OR, Cataldo R, Carassiti M, Gherardi S. Lightwand intubation using the Trachlight: a brief review of current knowledge. Can J Anesth 2001; 48: 592-9

5 Addas BM, Howes WJ, Hung OR. Light-guided tracheal puncture for percutaneous tracheostomy. Can J Anesth 2000; 47: 919-22

\section{Another report of obstruction of a heat and moisture exchange filter}

To the Editor:

I wish to congratulate Drs. Williams and Stacey on their report describing occlusion of a Pall heat and moisture exchange filter (HMEF) by pulmonary edema. ${ }^{1}$ I would like to report a similar incident involving a Pall BB25S HMEF.

A 54-yr-old, previously well man was undergoing debridement of a leg injury. The patient had been fasting since the time of his injury, $36 \mathrm{hr}$ previously. The anesthetic and procedure were uneventful until emergence, when the patient regurgitated a small amount of bile-stained fluid into the laryngeal mask. The fluid reached the HMEF, but it was not obviously heavily contaminated.

The patient developed signs of airway obstruction and auscultation revealed widespread inspiratory and expiratory rhonchi and coarse crepitations. The patient was treated for bronchospasm secondary to aspiration of gastric contents. There was no response to nebulized salbutamol, and the patient was then intubated because of increasing airway obstruction. Aliquots of adrenaline, 50-100 $\mu \mathrm{g}$, were given intravenously without effect, to a total of $400 \mu \mathrm{g}$.

The breathing circuit was then tested, and the HMEF was found to be occluded. The HMEF was changed and resolution of all obstruction occurred. The HMEF was examined. It was not obviously contaminated, but some regions of the HMEF were a faint yellow colour. The affected $\mathrm{HMEF}$ weighed $1 \mathrm{~g}$ more than a new filter. The patient did not sustain any longterm sequelae, and did not require any special care apart from supplementary oxygen for $12 \mathrm{hr}$.

Clifford J. Peady Fanzca

Garran, Australia

\section{Reference}

1 Williams DJ, Stacey MRW. Rapid and complete occlusion of a heat and moisture exchange filter by pulmonary edema (Clinical report). Can J Anesth 2002; 49: 126-31.

\section{The appropriateness of the pulmonary artery catheter in cardiovascular surgery}

To the Editor:

We read with interest the article "The appropriateness of the pulmonary artery catheter in cardiovascular surgery" from Jacka et al. ${ }^{1}$ At the Montreal Heart Institute, we use the PAC in all cardiac surgery cases and the transesophageal echocardiography (TEE) in more than half of our patients. Jacka et al. extrapolate from their results that a trial on the use of the PAC in stable patients during coronary revascularization is unethical. We disagree. Randomized controlled studies in the preoperative surgical setting have shown that a strategy based on the optimization of oxygen transport using the PAC can reduce mortality in noncardiac surgery ${ }^{2-4}$ and in cardiac surgery. ${ }^{5}$ It is also our experience that the PAC as an adjunct to improve oxygen transport is useful. The PAC can be used as either a diagnostic and prognostic tool. However, any type of monitoring can improve the prognostis only when the result is properly interpreted and effective treatment is introduced. Despite numerous retrospective reports of increased mortality associated with the use of the PAC, a recent randomized trial of 201 patients in the intensive care unit (ICU) has demonstrated that its use was not associated with an increase in mortality. $^{6}$ It is inappropriate to directly and totally attribute the excess mortality or morbidity to the PAC because it is used on sicker patients. Bearing in mind that the $\mathrm{PAC}$ is a diagnostic and monitoring tool and not a therapeutic device, it would be surprising that it (or TEE for this matter) would greatly reduce mortality. However, we believe that these devices used by trained individuals can alter the therapeutic approach. The PAC and TEE are particularly useful in the hemodynamically unstable patients. The PAC is useful in the postop ICU setting. During the off-working hours, the nonechographist intensivist or the resident on call can readily evaluate the hemodynamically unstable patients using all routine monitoring including the properly and electively placed PAC to assess response to therapy. Moreover, without the PAC, the continuous measure of pulmonary hypertension would not be 
easily made and treatment of this condition would not be promptly initiated.

Geneviève Arcand MD

André Denault MD FRCPC

Sylvain Bélisle MD FRCPC

Normand Tremblay MD FRCPC

Robert Blain MD FRCPC

Pierre Couture MD FRCPC

Peter Sheridan MD FRCPC

Philippe Sahab MD FRCPC

Norman Searle MD FRCPC

Jean Taillefer MD FRCPC

Montreal, Quebec

\section{References}

1 Jacka MJ, Cohen MM, To T, Devitt JH, Byrick R. The appropriateness of the pulmonary artery catheter in cardiovascular surgery. Can J Anesth 2002; 49: 276-82.

2 Shoemaker WC, Appel PL, Kram HB, Waxman K, Lee TS. Prospective trial of supranormal values of survivors as therapeutic goals in high-risk surgical patients. Chest 1988; 94: 1176-86.

3 Boyd O, Grounds RM, Bennett ED. A randomized clinical trial of the effect of deliberate perioperative increase of oxygen delivery on mortality in high-risk surgical patients. JAMA 1993; 270: 2699-707.

4 Wilson J, Woods I, Fawcett J, et al. Reducing the risk of major elective surgery: randomised controlled trial of preoperative optimisation of oxygen delivery. BMJ 1999; 318: 1099-103.

5 Polonen P, Ruokonen E, Hippelainen M, Poyhonen M, Takala J. A prospective, randomized study of goal- oriented hemodynamic therapy in cardiac surgical patients. Anesth Analg 2000; 90: 1052-9.

6 Rhodes A, Cusack RJ, Newman PJ, Grounds RM, Bennett ED. A randomised, controlled trial of the pulmonary artery catheter in critically ill patients. Intensive Care Med 2002; 28: 256-64.

\section{REPLY:}

We thank Dr. Arcand et al. for their letter and interest in this topic. ${ }^{1,2}$ We agree that the pulmonary artery catheter (PAC) is a diagnostic tool with no intrinsic therapentic value per se. Its value lies in the skill of its user and the purpose to which it is applied.

Evaluation of the PAC as Arcand et al. indicate, has been fraught with highly variable results both supporting and condemning its use. This illustrates well the difference between efficacy [use under optimal conditions such as in a randomized controlled trial (RCT)] and effectiveness (use under everyday circumstances). To explain this disparity, two major issues need to be considered.
First, evaluation of the PAC is difficult due to the extensive variability in the skill of users in everyday circumstances. Iberti et al. ${ }^{3}$ were first to document the difficulty that nearly $50 \%$ of clinicians had with estimation of the pulmonary artery occlusion pressure (PAOP), a finding which was also seen by Gnaegi et al. ${ }^{4}$ We also documented a disagreement rate of nearly $50 \%$ in $P A O P$ interpretation among anesthesiologists. ${ }^{5}$ Since the PAC is a technology, its optimal use requires the comprehension of multiple components of knowledge and their application. Assessment of the efficacy of the PAC using a RCT will require demonstrated standardization of the knowledge base necessary for effective use as a prereqwisite. While the RCT is an excellent tool for the evaluation of well-delineated interventions in specific circumstances, application of the RCT to assessment of the PAC represents a much larger undertaking than simply assigning patients at random to either receive the PAC or not, or to receive 'goal-directed' care or not.

Second, the RCT requires that clinical equipoise exists. In our study, clinicians were in extremely strong agreement, based on their experience, that the benefits of the PAC exceeded any potential harm in several scenarios, most of which involved pentricular impairment or unstable angina. They indicated strongly (90\%) that they would refuse to allow such patients to be assigned at random to receive the PAC or not. Clearly, clinical equipoise did not exist.

Clinicians agreed that the PAC was not indicated in the patient with preserved ventricular function or stable angina undergoing elective aortocoronary bypass or abdominal aneurysm resection. It has been suggested that this group of patients might be appropriate for a $R C T$ of the efficacy of the PAC in cardiac surgery. ${ }^{6}$ Aside from the fact that clinicians agreed that the PAC is unnecessary in these scenarios, mortality in elective cardiac surgery in this population ranges from 1-2\%.? Assuming that a shift of $0.5 \%$ in absolute mortality is clinically significant, approximately 2,000 patients in total would be needed to detect this difference. While possible in theory, this represents a labourious, time-consuming, multicentre undertaking, of which the most substantial benefit would be to demonstrate equivalence in outcome with and without the PAC, proving that which clinicians already know.

Instead of encouraging investigative effort to pursue RCTs that are likely to be unbelpful, we would recommend focusing on improving the knowledge base of capacities of the PAC by users. Moreover, the technologies in use deserving systematic evaluation and the related clinical questions are too numerous to perform RCTs to address each of them. What could be considered are surrogates of the RCT to answer relevant clinical questions, including 